\title{
Low redshift observational constraints on tachyon models of dark energy
}

\author{
Avinash Singh $^{a}$ Archana Sangwan $^{a}$ H. K. Jassal ${ }^{a}$ \\ ${ }^{a}$ Indian Institute of Science Education and Research Mohali, \\ SAS Nagar, Mohali 140306, Punjab, India. \\ E-mail: avinashsingh@iisermohali.ac.in, archanakumari@iisermohali.ac.in, \\ hkjassal@iisermohali.ac.in
}

\begin{abstract}
The background evolution of an accelerated, dark energy dominated universe is aptly described by non-canonical tachyon scalar field models. The accelerated expansion of the universe is determined by the choice of a suitable scalar field potential; in the case of a tachyon field, a 'runaway' potential. In the absence of a fundamental theory, dark energy properties are studied in a phenomenological approach. This includes determining the model parameters using observations and to probe the allowed deviation from the cosmological constant model. In this paper, we present constraints on tachyon scalar field parameters from low redshift data for two different scalar field potentials. These scalar field potentials have been crucial in tachyon dark energy studies. The datasets considered in this paper include the supernova type Ia data, independent measurements of the Hubble parameter and the Baryon Acoustic Oscillation data. In this paper, we present constraints on tachyon field parameters using these observations and their combination. A combination of the datasets indicates that those model parameters are preferred which emulate the cosmological constant model. The initial value of the scalar field, in the unit of the Hubble constant, is bounded from below and does not require fine-tuning at larger values.
\end{abstract}




\section{Contents}

1 Introduction 1

2 Solutions of Cosmological Equations 2

2.1 The Inverse Square Potential 3

2.2 The Exponential Potential 5

3 Observations 6

3.1 Baryon Acoustic Oscillation Data 6

3.2 Hubble parameter Data 7

3.3 Supernova Type Ia Data 8

4 Observational Constraints 9

4.1 Constraints on Inverse Square Potential 9

4.2 Constraints on Exponential Potential 11

5 Summary and Conclusions $\quad 14$

6 Acknowledgements 15

\section{Introduction}

Observations have established that more than two-thirds of the energy density of the universe is due to the contribution of dark energy [1-3]. Dark energy accounts for the observed late-time acceleration of the universe [4-6]. The nature of dark energy is, as yet, a mystery. To understand the nature of dark energy many models have been purposed, the simplest and the most favoured being the cosmological constant model [7,8]. For this component, the energy density remains a constant and the equation of state parameter is given by $w=-1$. Although this model is consistent with cosmological observations, the attempts to explain the cosmological constant as the energy density of vacuum suffers from the fine-tuning problem [8]. While the theoretical problem of fine-tuning remains, the cosmological constant model is the concordant model of dark energy.

Observations do not rule out models with an equation of state with $w \neq-1$, which is the property of other models such as the barotropic fluid models, canonical scalar field models, non-canonical scalar field models etc. The dynamical nature of the dark energy equation of state parameter is assumed by considering a functional form or parametrization of $w$. These parameterizations include those in which the equation of state parameter is a constant or is a function of time. The two key parameters are the present day value of the equation of state parameter and its derivatives. The simplest and most widely used prescription to model varying dark energy is the Chevallier-PolarskiLinder (CPL) parameterization $[9,10]$. This function has been widely employed in theoretical and observational studies of dark energy. Many other parameterizations have been described in [11-16]. The parameters are then constrained using different datasets, a few examples of such work are [1723].

Scalar fields are a well studied class of models for dark energy where the late time acceleration is achieved as the field evolves. The scalar field models include the canonical scalar fields such as quintessence fields and the non-canonical scalar fields such as tachyon fields and K-essence fields 
being a few examples. In scalar field models, the equation of state depends upon the functional form of the scalar field potential and on whether the kinetic energy term is sub-dominant or is the driving component of the evolution of the universe. In order to have an accelerated expansion, a slow rolling field is required. Although the scalar field models alleviate the fine-tuning problem that $\Lambda$ CDM model suffers from, to obtain the accelerated expansion in the recent era, they require tuning of their own. A detailed study for quintessence scalar field model is given in [24-33].

Tachyon scalar field belongs to the class of non-canonical scalar field models and has been studied in detail in [34]. Tachyon field arises naturally in string theory as a decay mode of D-branes [3537]. This field is a viable model of cosmology, and it has been shown that the tachyon scalar field can effectively explain dark energy. Apart from explaining accelerated expansion, an important property of this model is that its equation of state becomes dust like at early times, i.e., the equation of state parameter becomes zero in the past $[34,38,39]$. The dynamics of the universe in this case are driven by a 'runaway' potential $[40,41]$. The dark energy equation of state parameter is limited to the range $-1 \leq w_{\phi} \leq 0$ and hence phantom like equation of state is ruled out in this case. These considerations make this description of dark energy an interesting alternative to both fluid and canonical scalar field models. The tachyon models have also been extensively studied in the context of inflation [42-49]. Since tachyon scalar field shows dust like equation of state in its cosmic evolution, the 'tachyon dust' is considered as a potential candidate of combined dark energy and dark matter [36, 37, 50-54].

In this paper, we assume that the dark energy is described by a homogeneous tachyon field. We consider two models; one with an inverse power law potential and another with an exponential potential which have been the default potentials being used for studying tachyon field. We revisit the constraints on tachyon dark energy model with new data set of Baryon Acoustic Oscillations (BAO) [55-61], Supernova Type Ia (SN-Ia) [4-6, 62-68] and direct measurements of Hubble parameter $(\mathrm{H}(\mathrm{z}))$ [69-76]. We obtain stringent constraints on tachyon field parameters, by way of combining these datasets. Our motivation is to compare the constraints on the tachyon models from previous studies using the same datasets and to check if the non-canonical scalar field models prefer a different combination(s) of cosmological parameters. In this analysis, we have restricted ourselves to the low redshift datasets. The structure of the paper is as follows. In the next section 2, we discuss the background cosmology in the presence of a tachyon field and two different scalar potentials. The different low redshift observational datasets used to constrain the model parameters are discussed in section 3. In section 4, we discuss the results of the analysis for the models. We summarise and conclude the paper in section 5 .

\section{Solutions of Cosmological Equations}

The dynamics of the universe is governed by the Friedman equations which are given by

$$
\left(\frac{\dot{a}}{a}\right)^{2}=\frac{8 \pi G}{3} \rho, \quad \frac{\ddot{a}}{a}=-\frac{4 \pi G}{3}(\rho+3 P),
$$

where $\rho=\rho_{m}+\rho_{r}+\rho_{\phi}$. The quantities $\rho_{m}$ and $\rho_{r}$ are energy densities of non-relativistic matter(baryonic matter + dark matter ) and relativistic matter respectively whereas $\rho_{\phi}$ represents energy density of the tachyon field. The tachyon scalar field is described by the Lagrangian

$$
L=-V(\phi) \sqrt{1-\partial^{\mu} \phi \partial_{\mu} \phi},
$$

where $V(\phi)$ is an arbitrary potential. The energy density and pressure of tachyon field are

$$
\rho_{\phi}=\frac{V(\phi)}{\sqrt{1-\dot{\phi}^{2}}}, \quad P_{\phi}=-V(\phi) \sqrt{1-\dot{\phi}^{2}} .
$$



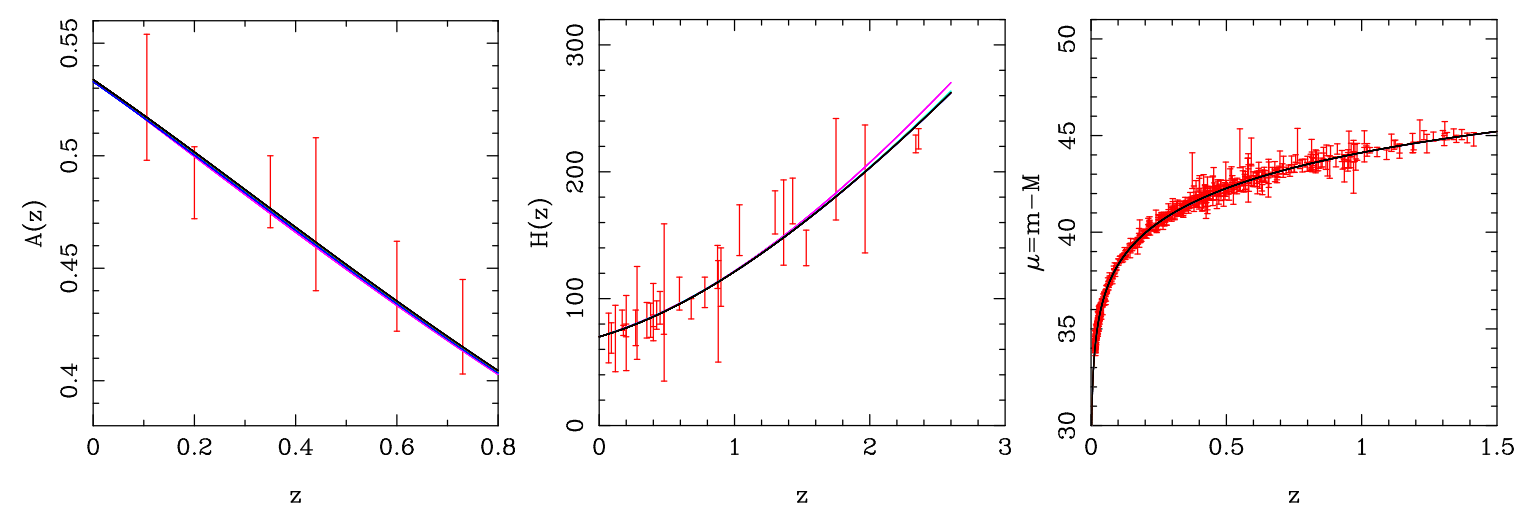

Figure 1. In this figure the acoustic parameter $A(z)$, the Hubble parameter $H(z)$ and the distance modulus $\mu(z)$ are shown in the plots from left to right respectively as a function of redshift $z$ for inverse square potential (2.5). The data points and error bars are taken from $[62,69,81]$. There are six very closely separated solid lines representing the model with inverse square potential in each plot for the values of the parameter $\phi_{0} H_{0}=2.0,3.0,4.0,5.0,6.0$ and 7.0. The values of other parameters $\Omega_{m 0}$ and $w_{\phi 0}$ are the corresponding best fit values taken from each row of the table 1 . There is a good agreement of the theoretical quantities with their observed values.

Therefore, the equation of state parameter of the tachyon field is $w_{\phi}=P_{\phi} / \rho_{\phi}=\dot{\phi}^{2}-1$. The dynamics of the scalar field is governed by the equation of motion for the scalar field

$$
\ddot{\phi}=-\left(1-\dot{\phi}^{2}\right)\left[3 H \dot{\phi}+\frac{1}{V(\phi)} \frac{d V}{d \phi}\right] .
$$

As $\dot{\phi}$ approaches \pm 1 , the equation of state becomes dust like, and the quantity $\ddot{\phi}$ goes to zero. Therefore, the equation of state remains dust like for a long time. The cosmological evolution in this model depends on the choice of potential. We consider two runaway potentials which have been employed to study tachyon dynamics. The runaway potential naturally arises in string theory and M-theory, and they are capable of generating the late time accelerated expansion of the universe [77-80]. The background cosmology in the presence of two different tachyon scalar field potentials is summarized below.

\subsection{The Inverse Square Potential}

A potential which describes a tachyon scalar field model of dark energy is given as

$$
V(\phi)=\frac{n}{4 \pi G}\left(1-\frac{2}{3 n}\right)^{1 / 2} \phi^{-2},
$$

where the real number $n$ determines the amplitude of the potential. The inverse square potential leads to a cosmological evolution of the form $a=t^{n}$ [38]. Cosmological dynamics of tachyon scalar field dark energy with this potential have been studied in [34], and the stability analysis of this potential has been done in [39-41]. The cosmological dynamics depends on the quantity $\lambda=-M_{n} V^{-3 / 2} d V / d \phi$ which is a constant for this potential. With the slow-rolling condition, this leads to a stable critical fixed point for this potential which can generate a late time accelerated expansion (with $n>1$ ). This fixed point is an attractor which leads to $\Omega_{\phi}=1$ and the equation of state parameter $w_{\phi}=2 / 3 n-1$ asymptotically. There still remains the requirement of a tuning, which is needed for a sufficient acceleration at the present time [39, 40]. 

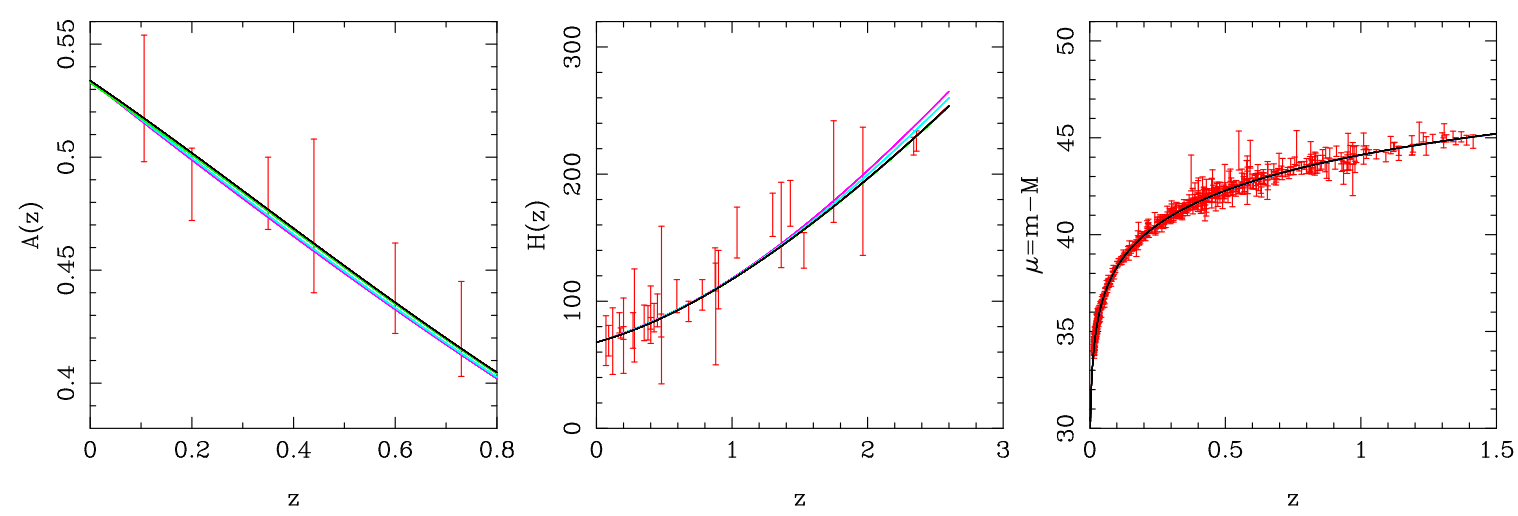

Figure 2. The figure shows plots of the acoustic parameter $A(z)$, the Hubble parameter $H(z)$ and the distance modulus $\mu(z)$ as a function of redshift $z$ for the exponential potential (2.14). The data points and error bars are taken from $[62,69,81]$. There are six very closely separated theoretical solid lines representing tachyon dark energy model with exponential potential for $\phi_{0} H_{0}=0.08,0.09,0.1,0.3,0.5$ and 0.7 . We have fixed the parameter $\phi_{0} / \phi_{a}=0.1$ and the values of other parameters $\Omega_{m 0}$ and $w_{\phi 0}$ are the corresponding best fit values taken from each row of the table 2 .

To numerically solve the cosmological equations, we transform the above equations by introducing the following dimensionless variables

$$
\begin{aligned}
& y=\frac{a(t)}{a\left(t_{\text {in }}\right)}, \psi=\frac{\phi(t)}{\phi\left(t_{\text {in }}\right)}, \\
& x=H_{\text {in }},
\end{aligned}
$$

here ' $t_{i n}$ ' represents the initial time. The equations can then be written as

$$
\begin{aligned}
y^{\prime} & =y\left[\Omega_{m, i n} y^{-3}+\Omega_{r, i n} y^{-4}+\frac{\Omega_{\phi, i n} \sqrt{-w_{\phi, i n}}}{\psi^{2} \sqrt{1-\phi_{i n}^{2} H_{i n}^{2} \psi^{\prime 2}}}\right]^{1 / 2}, \\
\psi^{\prime \prime} & =\left(1-\phi_{i n}^{2} H_{i n}^{2} \psi^{\prime 2}\right)\left[\frac{2}{\phi_{i n}^{2} H_{i n}^{2} \psi}-3 \psi^{\prime} \frac{y^{\prime}}{y}\right],
\end{aligned}
$$

The prime on superscript denotes derivative with respect to $x=H_{\text {in }}$, and different $\Omega$ 's are dimensionless density parameters defined as the ratio of the density of the relevant component and critical density $\rho_{c r}=\frac{3 H_{0}^{2}}{8 \pi G}$. Here, we have assumed the universe to be spatially flat and hence $\Omega_{\text {total }}=$ $\Omega_{m, i n}+\Omega_{r, i n}+\Omega_{\phi, i n}=1$.

We integrate the equations numerically from the present time $\left(t_{i n}=t_{0}\right)$ to early times, and $\Omega_{m 0}$, $\phi_{0} H_{0}$ and $\dot{\phi}_{0}$ or $w_{\phi 0}$ are the parameters which are varied. The amplitude of the potential can be constrained by using the relation

$$
\frac{2 n}{3}\left(1-\frac{2}{3 n}\right)^{1 / 2}=\Omega_{\phi 0} \phi_{0}^{2} H_{0}^{2} \sqrt{-w_{\phi 0}} .
$$

To calculate the value of $n$ from above, the equation we need to solve is the polynomial equation

$$
12 n^{3}-8 n^{2}-27 q^{2} n=0,
$$

where $q=\Omega_{\phi 0} \phi_{0}^{2} H_{0}^{2} \sqrt{-w_{\phi 0}}$ is a positive number. The solution of equation (2.9) for accelerated expansion $(n>1)$ is

$$
n=\frac{1}{3}+\frac{1}{6} \sqrt{4+(9 q)^{2}}
$$



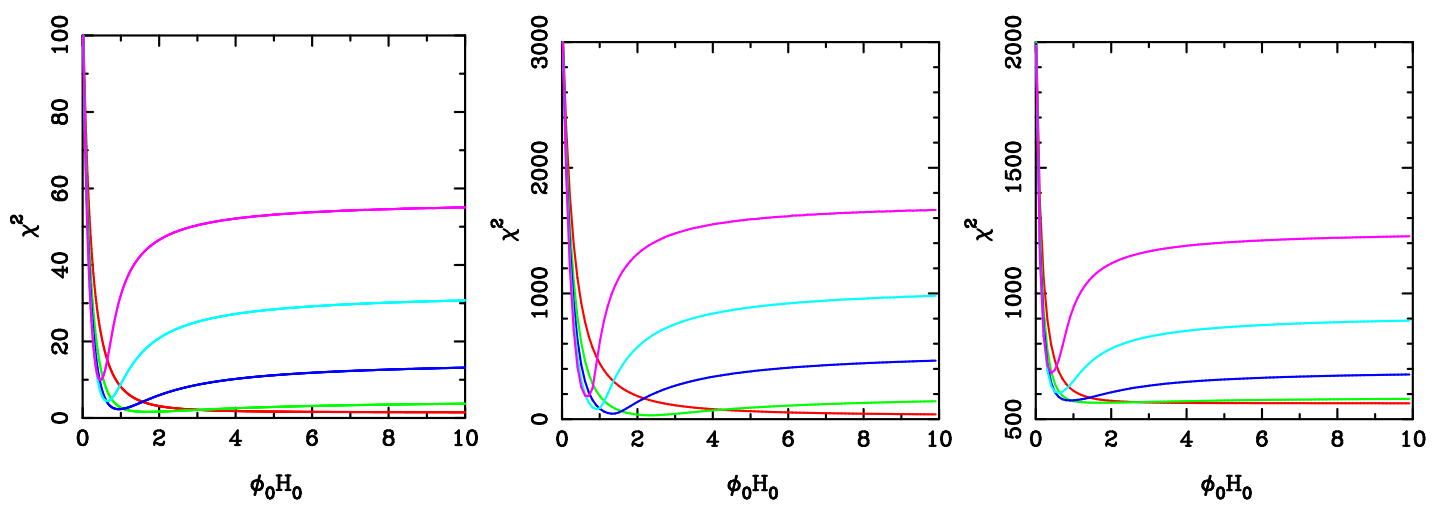

Figure 3. Going from left to right, the plots show $\chi^{2}$ as a function of $\phi_{0} H_{0}$ for BAO, $H(z)$ and SN-Ia data respectively. Here have fixed $\Omega_{m 0}=0.285$ whereas the red, green, blue, sky-blue and pink lines represent the value of $w_{\phi 0}$ to be $-1.0,-0.95,-0.90,-0.85$ and -0.80 respectively.

with the condition that $q>\frac{2 \sqrt{3}}{9}$. The value of the present day radiation density parameter $\Omega_{r 0}$ is [82]

$$
\Omega_{r 0}=\frac{\Omega_{m 0}}{1+z_{e q}},
$$

where $z_{e q}=2.5 \times 10^{4} \Omega_{m 0} h^{2}\left(T_{c m b} / 2.7 \mathrm{~K}\right)^{-4}, T_{c m b}=2.7255 \mathrm{~K}$.

The initial conditions for the numerical solutions are

$$
y_{0}=1, \psi_{0}=1
$$

and $\psi_{0}^{\prime}$ can be calculated using relation

$$
\psi^{\prime}=\frac{\dot{\phi}}{\phi_{0} H_{0}}=\frac{\sqrt{1+w_{\phi}}}{\phi_{0} H_{0}} .
$$

\subsection{The Exponential Potential}

The exponential potential for tachyon scalar field dark energy is given by

$$
V(\phi)=V_{a} \exp \left(-\phi / \phi_{a}\right)
$$

where amplitude $V_{a}$ and $\phi_{a}$ are the scalar field parameters. Cosmological dynamics with this potential have also been studied in [34], and the stability analysis of this potential has been done in [39-41]. For this potential, $\lambda \rightarrow \infty$ as $\phi \rightarrow \infty$. This is a fixed point for which $\Omega_{\phi} \simeq 0$ and a dust like equation of state. Since $\lambda$ changes dynamically [40], the universe goes to a temporary accelerated phase for $\lambda \lesssim 1$ and enters a decelerated phase for $\lambda \gg 1$. In other words, the present day acceleration is temporary, and the universe enters a phase of decelerated expansion once again. This evolution of the universe, therefore, avoids the future event horizon problem.

Introducing the same dimensionless variables as introduced in the last subsection, we can transform the required equations as

$$
\begin{aligned}
y^{\prime} & =y\left[\Omega_{m, i n} y^{-3}+\Omega_{r, i n} y^{-4}+\frac{\Omega_{\phi, i n} \sqrt{-w_{\phi, i n}} e^{\frac{\phi_{i n}}{\phi_{a}}}(1-\psi)}{\sqrt{1-\phi_{i n}^{2} H_{i n}^{2} \psi^{\prime 2}}}\right]^{1 / 2}, \\
\psi^{\prime \prime} & =\left(1-\phi_{i n}^{2} H_{i n}^{2} \psi^{\prime 2}\right)\left[\frac{\phi_{i n} / \phi_{a}}{\phi_{i n}^{2} H_{i n}^{2}}-3 \psi^{\prime} \frac{y^{\prime}}{y}\right]
\end{aligned}
$$



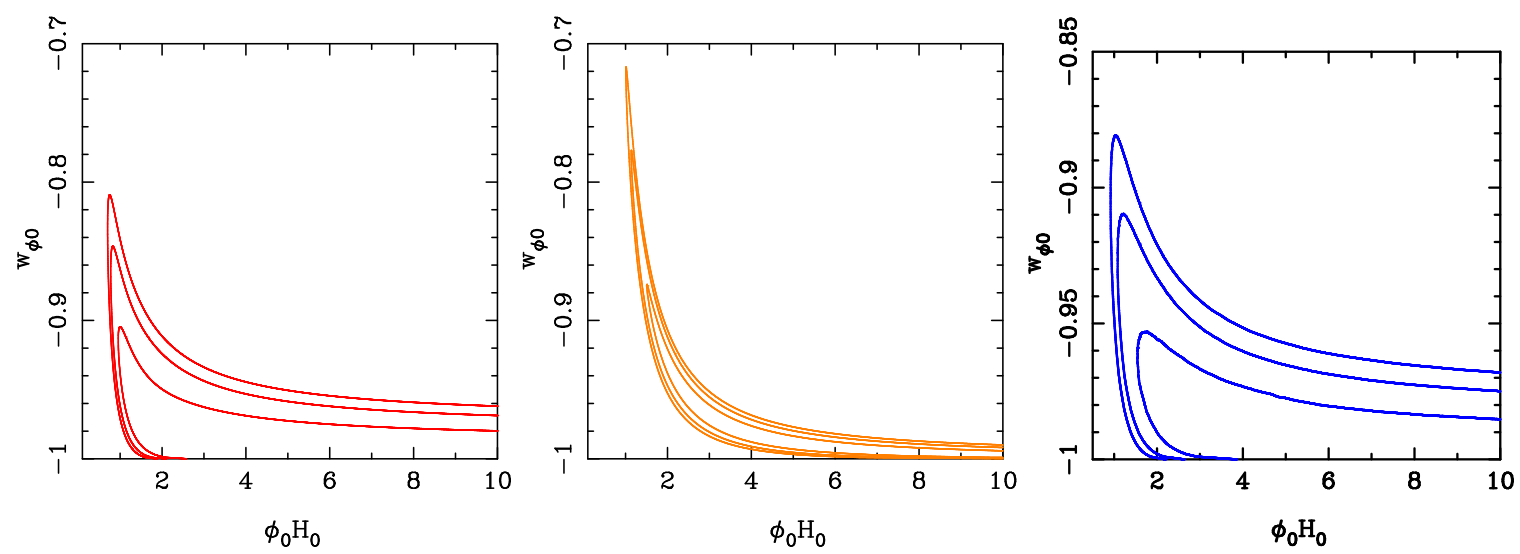

Figure 4. The figure shows $1 \sigma, 2 \sigma$ and $3 \sigma$ confidence contours between $w_{\phi 0}$ and $\phi_{0} H_{0}$ for $\Omega_{m 0}=0.285$ for $\mathrm{BAO}, H(z)$ and $\mathrm{SN}-\mathrm{Ia}$ data in plots from left to right respectively.

We, therefore, have three model parameters $\phi_{0} H_{0}, \phi_{0} / \phi_{a}$ and $\dot{\phi}_{0}$ or $w_{\phi 0}$ to constrain. Apart from these parameters, there are cosmological parameters $\Omega_{m 0}$ and $H_{0}$. In this case, the amplitude of potential can be calculated by the relation

$$
\frac{8 \pi G}{3 H_{0}^{2}} V_{a}=\Omega_{\phi 0} e^{\phi_{0} / \phi_{a}} \sqrt{-w_{\phi 0}},
$$

Structure of these equations suggest that $-1 \leq w_{\phi 0}=\phi_{0}^{2} H_{0}^{2} \psi_{0}^{\prime 2}-1<0$. For this potential, we also use the same initial conditions given in equation (2.12) and (2.13).

\section{Observations}

\subsection{Baryon Acoustic Oscillation Data}

Baryon Acoustic Oscillations (BAO) [55-61] observations are the measurement of baryon oscillation feature in the correlation function of large scale structure (LSS). This feature is the result of acoustic waves in the pre-recombination baryon-photon plasma caused by opposing forces of gravity and radiation. These acoustic waves left an imprint on the baryonic clustering in the universe and gave rise to the baryon acoustic oscillation peaks. The characteristic angular scale of the acoustic peak is $\theta_{A}=r_{s}\left(z_{d}\right) / D_{V}(z)$, where $r_{s}$ is sound horizon at drag epoch $z_{d}$, which is given by

$$
r_{s}\left(z_{d}\right)=\int_{z_{d}}^{\infty} \frac{c_{s}(z)}{H(z)} d z
$$

and $D_{V}$ is effective distance ratio, and it can be calculated using the angular diameter distance $D_{A}(z)$ as follows

$$
D_{V}(z)=\left[(1+z)^{2} D_{A}(z)^{2} \frac{c z}{H(z)}\right]^{1 / 3} .
$$

We use the BAO data from Baryon Oscillation Spectroscopic Survey (BOSS) DR12 [83] which provides 6 data points (see table-7 of Alam et al.) at redshifts $z=0.38,0.51,0.61$ in terms of $H(z) r_{s}\left(z_{d}\right) / r_{s, f i d}$ and $D_{M}(z) r_{s, f i d} / r_{s}\left(z_{d}\right)$ where $r_{s, f i d}=147.78 \mathrm{Mpc}$ and $D_{M}(z)=(1+z) D_{A}(z)$ is the comoving angular diameter distance. The sound horizon $r_{s}\left(z_{d}\right)$ given by [84]

$$
r_{s}\left(z_{d}\right)=\frac{55.154 \exp \left[-72.3\left(\omega_{v}+0.0006\right)^{2}\right]}{\omega_{b}^{0.12807} \omega_{c b}^{0.25351}} \mathrm{Mpc}
$$



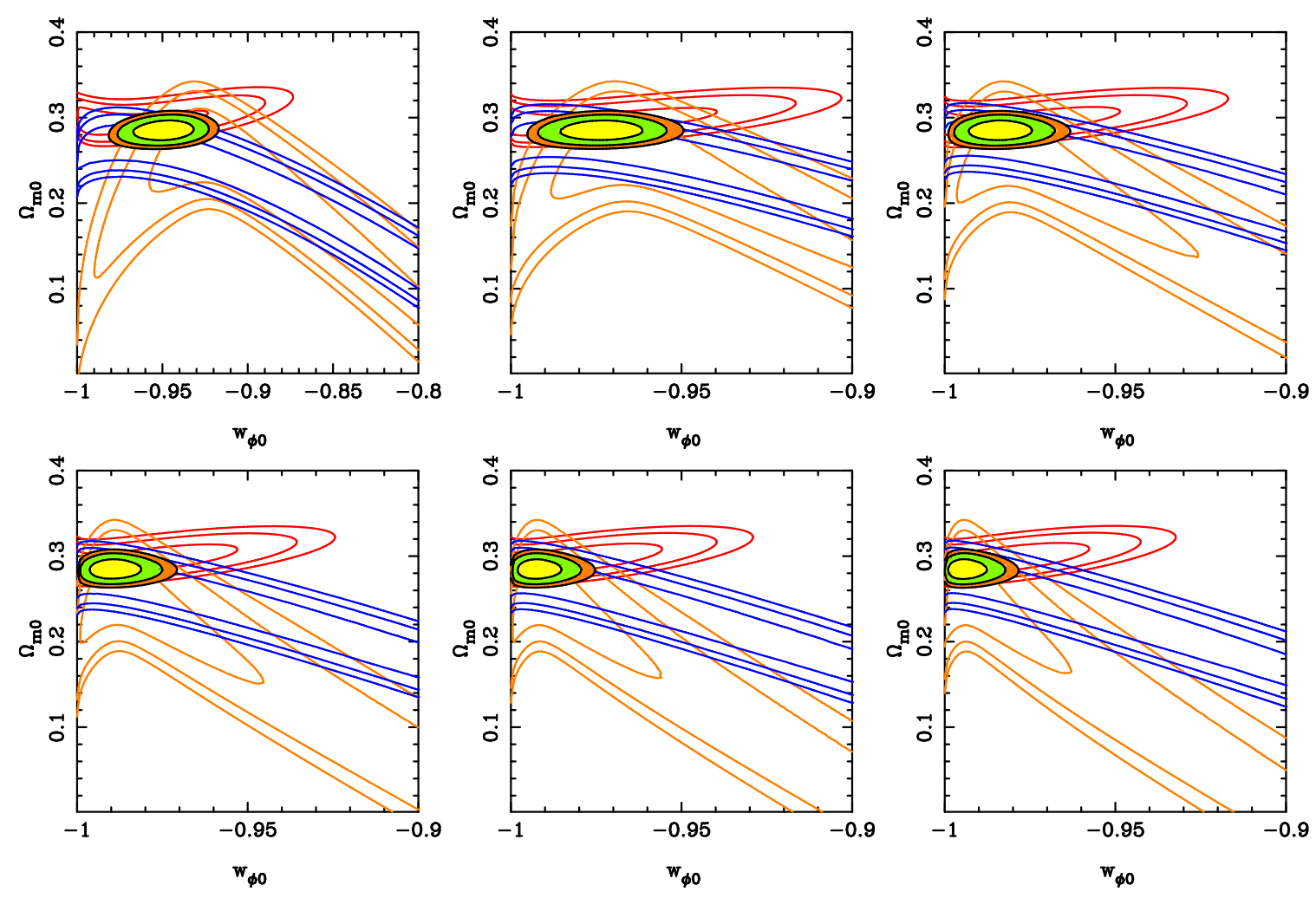

Figure 5. The plots show contours in the $\Omega_{m}-w_{\phi}$ plane, for constant $\phi_{0} H_{0}$ for all the three and combined datasets for the inverse square potential. Contours in red, orange and blue are for BAO, $H(z)$ and SN-Ia data respectively. Black contours filled with colours represent combined constraints. The value of parameter $\phi_{0} H_{0}=2.0,3.0,4.0$ for plots in row-1 and 5.0, 6.0, 7.0 for plots in row-2 from left to right respectively.

where $\omega_{v}=\Omega_{v} h^{2}=0.0107\left(\sum m_{v} / 1.0 e V\right), \omega_{b}=\Omega_{b} h^{2}$ and $\omega_{c b}=\Omega_{m} h^{2}-\omega_{v}$. Symbols $\Omega_{v}, \Omega_{b}$ and $\Omega_{m}$ represent density parameters of neutrinos, baryons and non-relativistic matter (baryonic matter + dark matter). We set mass of neutrinos $\sum m_{v}=0.06 \mathrm{eV}$ and $\Omega_{b} h^{2}=0.02225$ with $h=0.676$.

We also use BAO data from LOWZ and CMASS at redshift $z=0.32$ and 0.59 as given in reference [85]. Here $r_{s, f i d}=147.66 \mathrm{Mpc}$ and the approximation for $r_{s}\left(z_{d}\right)$ is the same as shown in equation (3.3). We also use older BAO data from 6dFGS, SDSS DR7 and WiggleZ at redshifts $z=0.106,0.2,0.35,0.44,0.6$ and 0.73 . These are listed in table-3 of [81] in term of the acoustic parameter. The acoustic parameter [86] is

$$
A(z)=\frac{100 D_{A} \sqrt{\Omega_{m} h^{2}}}{c z} .
$$

Here $c$ is the speed of light in vacuum.

\subsection{Hubble parameter Data}

Hubble parameter data set [69-76] consists of values of Hubble parameters $H(z)$ at different redshifts and associated errors in the corresponding measurement. We use $H(z)$ dataset compiled and listed in table-1 of [69]. The table contains values of the Hubble parameters $H(z)$ at 38 different redshifts with associated errors in measurement and corresponding references up to redshift $z=2.3$. Out of 38 we use only 32 points as we do not consider three data points taken from Alam et. al.(2016) 


\begin{tabular}{|c|c|c|c|c|}
\hline$\phi_{0} H_{0}$ & $\chi_{\text {min }}^{2}$ & $\Omega_{m 0}$ & $w_{\phi 0}$ & $n$ \\
\hline 2.0 & 596.145 & $0.285_{-0.022}^{+0.023}$ & $-0.950_{-0.031}^{+0.033}$ & {$[4.323,4.726]$} \\
\hline 3.0 & 592.045 & $0.285_{-0.021}^{+0.023}$ & $-0.973_{-0.022}^{+0.023}$ & {$[9.445,10.250]$} \\
\hline 4.0 & 590.944 & $0.284_{-0.021}^{+0.024}$ & $-0.984_{-0.015}^{+0.021}$ & {$[16.635,18.016]$} \\
\hline 5.0 & 590.515 & $0.285_{-0.022}^{+0.023}$ & $-0.990_{-0.009}^{+0.019}$ & {$[25.906,27.959]$} \\
\hline 6.0 & 590.335 & $0.285_{-0.022}^{+0.023}$ & $-0.993_{-0.007}^{+0.017}$ & {$[37.252,40.133]$} \\
\hline 7.0 & 590.285 & $0.285_{-0.022}^{+0.023}$ & $-0.995_{-0.005}^{+0.016}$ & {$[50.659,54.504]$} \\
\hline
\end{tabular}

Table 1. The table lists the best fit values of $\Omega_{m 0}$ and $w_{\phi 0}$ along with their $3 \sigma$ confidence range for different values of $\phi_{0} H_{0}$ for inverse square potent for combined data $(\mathrm{BAO}+\mathrm{H}(\mathrm{z})+\mathrm{SN}-\mathrm{Ia})$. In the second column minimum value of corresponding $\chi_{\min }^{2}$ have been shown. In the last column, we have shown the $3 \sigma$ allowed range of ' $n$ ', calculated from equation (2.10) considering $3 \sigma$ confidence range of $\Omega_{m 0}$ and $w_{\phi 0}$.

at redshifts $z=0.38,0.51,0.61$ and three data points taken from Black et. al.(2012) at redshifts $z=0.44,0.6,0.73$ as we include these data points in our BAO dataset. Hubble parameter can be computed from the Friedmann equation and is given by

$$
H(z)=H_{0}\left[\Omega_{m 0}(1+z)^{3}+\Omega_{r 0}(1+z)^{4}+\Omega_{\phi}\right]^{1 / 2},
$$

where $H_{0}$ is the present value of the Hubble parameter.

\subsection{Supernova Type Ia Data}

The third dataset we use for our analysis is observations of supernovae type Ia [4-6, 62-68], which is supernova explosion of a white dwarf star accreting mass from its binary companion and hence crosses the Chandrasekhar limit. When a white dwarf star crosses the Chandrasekhar mass limit of $1.4 M_{\odot}$, it explodes: this is a Supernova explosion of Type-Ia. Luminosity distance of Supernova-Ia which occurred at redshift $z$ is given by the relation

$$
d_{L}(z)=\frac{c}{H_{0}}(1+z) \int_{0}^{z} \frac{1}{E(z)} d z .
$$

where function $E(z)=H(z) / H_{0}$. In SN-Ia data, we have distance moduli of 580 supernovae up to redshifts $z=1.414$ along with their associated observational error [62]. The theoretical values of distance modulus can be calculated using luminosity distance as

$$
\mu=5 \log \left(d_{L}\right)-5,
$$



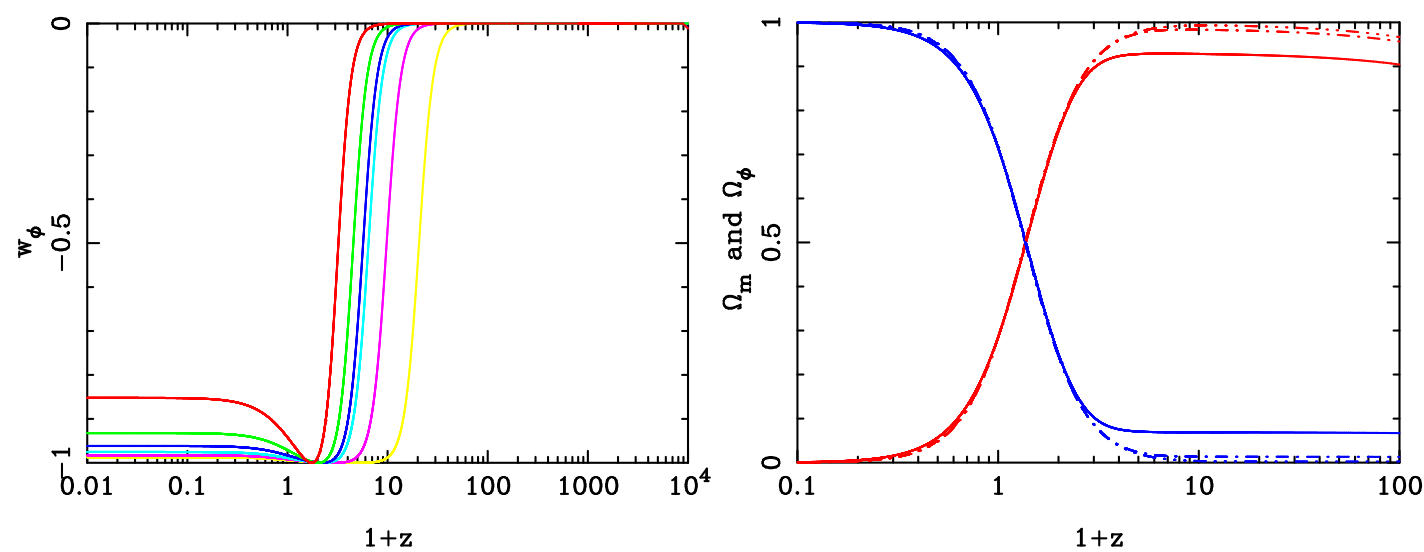

Figure 6. The plot on the left shows the evolution of equation of state $w_{\phi}$ with redshift for inverse square potential. Red, green, blue, sky-blue, yellow and pink colours represent value of $\phi_{0} H_{0}=2.0,3.0,4.0,5.0,6.0$ and 7.0. The plot on the right shows the evolution of $\Omega_{m}$ (red curves) and $\Omega_{\phi}$ (blue curves) with redshift. The solid, dash-dot and dashed-dot-dot-dot lines represent the value of $\phi_{0} H_{0}=2.0,4.0$ and 6.0 respectively. The value of parameter $w_{\phi 0}$ and $\Omega_{m 0}$ are the best fit values taken from table 1 for the corresponding value of $\phi_{0} H_{0}$.

here $d_{L}$ is in the unit of $10 p c$ and $\mu=m-M$ is the distance modulus, $m$ and $M$ are the apparent and absolute magnitude respectively of the supernova.

\section{Observational Constraints}

We do the standard $\chi^{2}$ analysis to constrain parameters for the tachyon dark energy. Value of $\chi_{B A O}^{2}$ for the Baryon Acoustic Oscillation data is the sum of $\chi^{2}$ over all redshifts given in subsection 3.1. We calculated $\chi^{2}$ for DR12 data using the expression given by

$$
\chi^{2}=\sum_{i, j=1}^{N}\left[X_{t h, i}-X_{o b s, i}\right] C_{i, j}^{-1}\left[X_{t h, j}-X_{o b s, j}\right]
$$

where $N$ is the number of data points in BAO dataset, $X_{t h}$ is a vector of the theoretical value of corresponding observable and $X_{o b s}$ is a vector of the observational values. We employ the covariance matrix $C_{i j}$ taken from the online files of Alem et al. (2017) and Chi-Hsun et al. (2017). Value of $\chi^{2}$ for older BAO data (BAO data from 6dFGS, SDSS DR7 and WiggleZ), H(z) data and SN-Ia data is calculated using

$$
\chi_{\text {oderBAO/Hz/SN }}^{2}=\sum_{i=1}^{N}\left(\frac{O_{D}\left(z_{i}\right)-O_{M}\left(z_{i}, \mathbf{p}\right)}{\sigma_{i}}\right)^{2},
$$

Here $O_{D}\left(z_{i}\right)$ is the theoretical value of the observable at redshift $z_{i}$, and $O_{M}\left(z_{i}, \mathbf{p}\right)$ is its value for model at redshift $z_{i}$ with the set of parameters $\mathbf{p}$. The quantity $\sigma_{i}$ is the error in the measurement of the observable $O_{D}\left(z_{i}\right)$. Here observable ' $O$ ' is the acoustic parameter $A(z)$ for the older BAO data, Hubble parameter for the $\mathrm{H}(\mathrm{z})$ data and distance modulus $\mu(z)$ for the SN-Ia data. We then find the maximum likelihood $\left(e^{-\chi_{t o t}^{2}}\right)$ of the parameter space by minimizing $\chi_{t o t}^{2}=\chi_{B A O}^{2}+\chi_{H z}^{2}+\chi_{S N}^{2}$.

\subsection{Constraints on Inverse Square Potential}

As mentioned in section 2.1, we constrain three parameters, $\Omega_{m 0}, w_{\phi 0}$ and $\phi_{0} H_{0}$ for the potential. Since only the square of the quantity $\phi_{0} H_{0}$ appears in the equations, we need to consider only one 
of the two, positive or negative branches. There is a degeneracy between parameters $w_{\phi 0}$ and $\phi_{0} H_{0}$; these parameters are correlated.

In figure 1, we plot the acoustic parameter $A(z)$ obtained from the BAO data from $6 \mathrm{dFGS}$, SDSS DR7 and WiggleZ [81]. In the plot on middle and on the right we have shown the Hubble parameter $H(z)$ and the distance modulus $\mu(z)$ as a function of $z$ respectively. Data points and error bars are taken from $[62,69]$. There are six (overlapping) theoretical solid lines in each of these plots representing inverse square potential (2.5). To draw these theoretical lines we have taken the best fit value of parameters $\phi_{0} H_{0}, \Omega_{m 0}$ and $w_{\phi 0}$ from each row of table 1 . We can see that there is a good agreement of the theoretical curves with data.

The values of $\chi^{2}$ vs $\phi_{0} H_{0}$ for $\Omega_{m 0}=0.285$ are plotted in figure 3. The five different colours represent different values of $w_{\phi 0}$ from -1.0 to -0.80 in the steps of 0.05 . We can see that if $w_{\phi 0}$ is close to -1.0 (red and green curves) all larger value of $\phi_{0} H_{0}$ are allowed. If we fix $w_{\phi 0}$ to a value away form -1.0 , we can get a minimum in $\chi^{2}$ curves and fixing this parameter is equivalent to fixing $\dot{\phi}_{0}$ as $w_{\phi}=\dot{\phi}^{2}-1$. Since we are interested in constraining $\Omega_{m 0}$ and $w_{\phi 0}$, we choose to fix $\phi_{0} H_{0}$. Degeneracy between these parameters can also be seen in figure 4 where we have shown $1 \sigma, 2 \sigma$ and $3 \sigma$ contours in the $w_{\phi 0}-\phi_{0} H_{0}$ plane for the three datasets. After marginalizing over $\Omega_{m 0}$, we find that $\phi_{0} H_{0} \geq 0.775$ at $3 \sigma$ confidence level using combined data. It can be clearly seen that there is a bound on the lower value of $\phi_{0} H_{0}$ but not on its upper value and hence we can not marginalize over $\phi_{0} H_{0}$. We, therefore, have constrained the parameter space of $\Omega_{m 0}-w_{\phi 0}$ and shown its variation with $\phi_{0} H_{0}$ in figure 5 .

For each of these contours, we have fixed the value of $\phi_{0} H_{0}$. The most stringent constraints come from the BAO data, and combined constraints limit the parameter space to a very small range. Value of $\Omega_{m 0}$ is well constrained by combined dataset at $0.285_{-0.022}^{+0.023}$ with $3 \sigma$ confidence, and this remains almost same with variation in parameter $\phi_{0} H_{0}$. However, the constraint on $w_{\phi_{0}}$ depends on the value of $\phi_{0} H_{0}$. As we increase the value of $\phi_{0} H_{0}$, all the three datasets prefer a value of $w_{\phi 0}$ close to -1 . In table 1 we have shown, the minimum value of $\chi^{2}$ for a fixed value of $\phi_{0} H_{0}$ and the best fit value of parameters $\Omega_{m 0}$ and $w_{\phi 0}$ with the $3 \sigma$ confidence limit for combined data. We started with $\phi_{0} H_{0}=2.0$ and increased its value in unit step. Here we can see that minimum value of $\chi^{2}$ saturates for a larger value of $\phi_{0} H_{0}$, and so does the parameter $\Omega_{m 0}$. In this background cosmological model, we can tune the parameter $\phi_{0} H_{0}$ to be very close to $w_{\phi}=-1.0$. In the strict sense, it is not possible to constrain $\phi_{0} H_{0}$ using these background data. A large range of values of $\phi_{0} H_{0}$ are acceptable as the background data only put a lower bound on its value. In the last column of table 1 , we have shown the $3 \sigma$ allowed range of ' $n$ ' computed from the equation (2.10) considering the $3 \sigma$ confidence range of $\Omega_{m 0}$ and $w_{\phi 0}$. From equation (2.8), it is clear that the amplitude of the tachyon potential and constant ' $q$ ' are proportional to the value of $\phi_{0}^{2} H_{0}^{2}$, as can be seen in the equation (2.8). This is the reason, the allowed value of ' $n$ ' also increases with it. Since the universe expands like $a \propto t^{n}$ for a given ' $n$ ', for a larger value of $\phi_{0} H_{0}$ the accelerated expansion is faster in dark energy dominated era. We find that for this model, the transition redshift is between $0.61 \lesssim z_{a c c} \lesssim 0.80$.

The evolution of the matter density parameter $\Omega_{m}(z)$ (red curves) and the dark energy density parameter $\Omega_{\phi}(z)$ (blue curves) are shown in the plot on the right in figure 6 . We can see that even in the matter dominated era, dark energy contributes significantly to the energy budget. For smaller values of $\phi_{0} H_{0}$, the contribution of dark energy, in the matter dominated era, is larger than it is for larger value of this parameter. Here it should be noted that parameter $\phi_{0} H_{0}$ and $w_{\phi 0}$ are correlated. As we increase the value of $\phi_{0} H_{0}$ matter approaches complete domination for large redshift.

The evolution of the equation of state of the dark energy $w_{\phi}$ is shown in the plot on the left in figure 6. In the matter dominated era and before it, the equation of state of tachyon dark energy is just like that of dust. After that, it starts evolving and make a sharp transition towards smaller value 

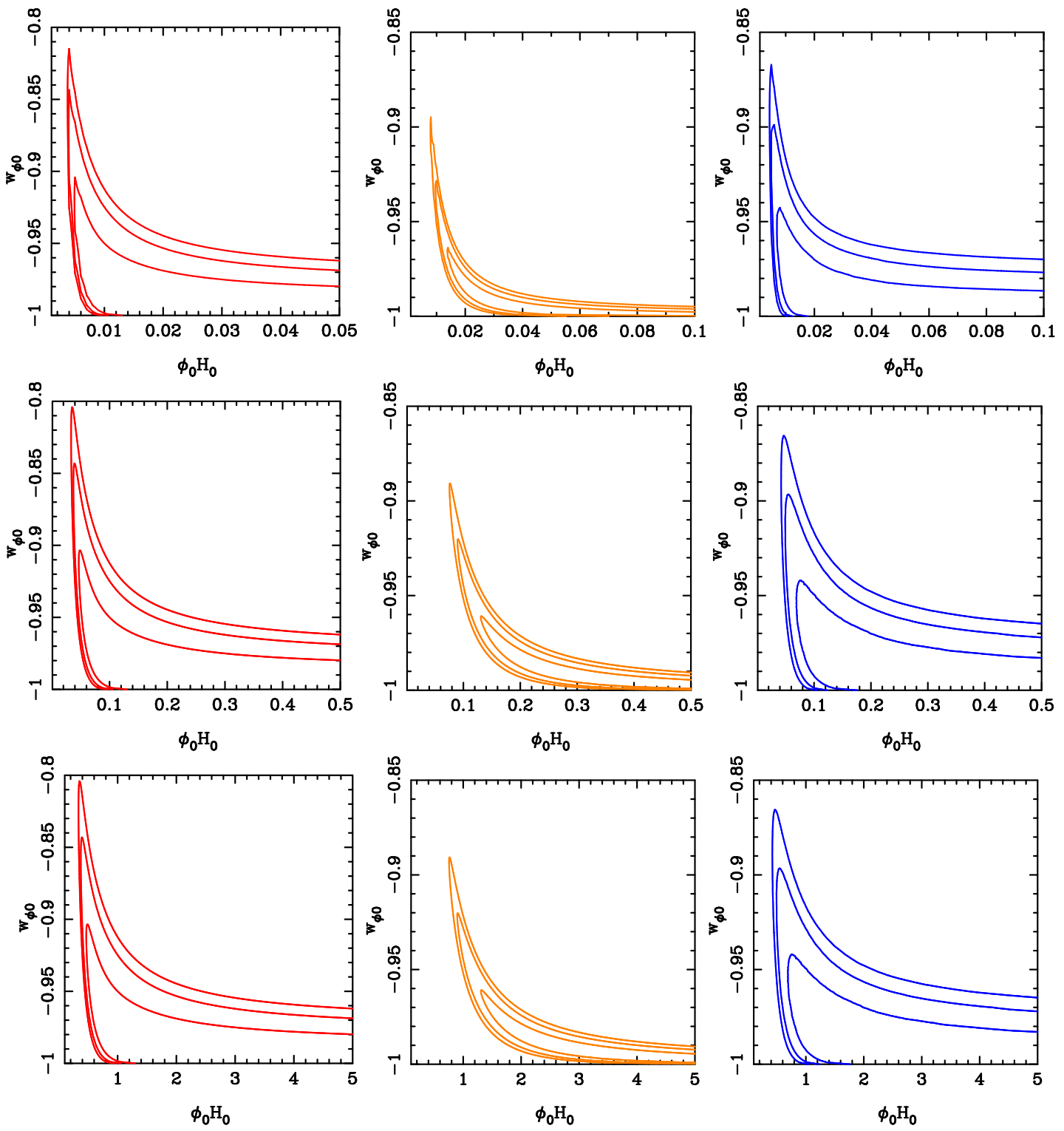

Figure 7. The figure shows we $1 \sigma, 2 \sigma$ and $3 \sigma$ confidence contours on $w_{\phi 0}-\phi_{0} H_{0}$ plane for exponential potential. Red, orange and blue colours represent BAO, Hz and SN-Ia data respectively. First, second and third rows are for $\phi_{0} / \phi_{a}=0.01,0.1$ and 1.0. For all these plots we have set $\Omega_{m 0}=0.285$.

than its present value $w_{\phi 0}$ then rises again. For a given value of $\phi_{0} H_{0}$ it maintains a constant value in the future. For the larger values of $\phi_{0} H_{0}$, this constant value for future evolution is closer to -1.0 as a larger value of $\phi_{0} H_{0}$ prefers a cosmological constant like behaviour.

\subsection{Constraints on Exponential Potential}

For the exponential potential, we need to constrain parameters $\phi_{0} H_{0}, \phi_{0} / \phi_{a}, w_{\phi 0}$ and $\Omega_{m 0}$. Rewriting the potential as $V=e^{\ln \left(V_{a}\right)-\frac{\phi}{\phi_{a}}}$, we see that there is an explicit degeneracy between $V_{a}$ and $\phi_{i n}$, i.e., a change in $V_{a}$ and the corresponding change in $\phi_{i n}$ leads to the same $V_{i n}$. Since we have replaced $V_{a}$ by the other parameters shown in equation (2.16), this degeneracy reflects in degeneracy between $\phi_{0} / \phi_{a}$ and $\phi_{0} H_{0}$. 

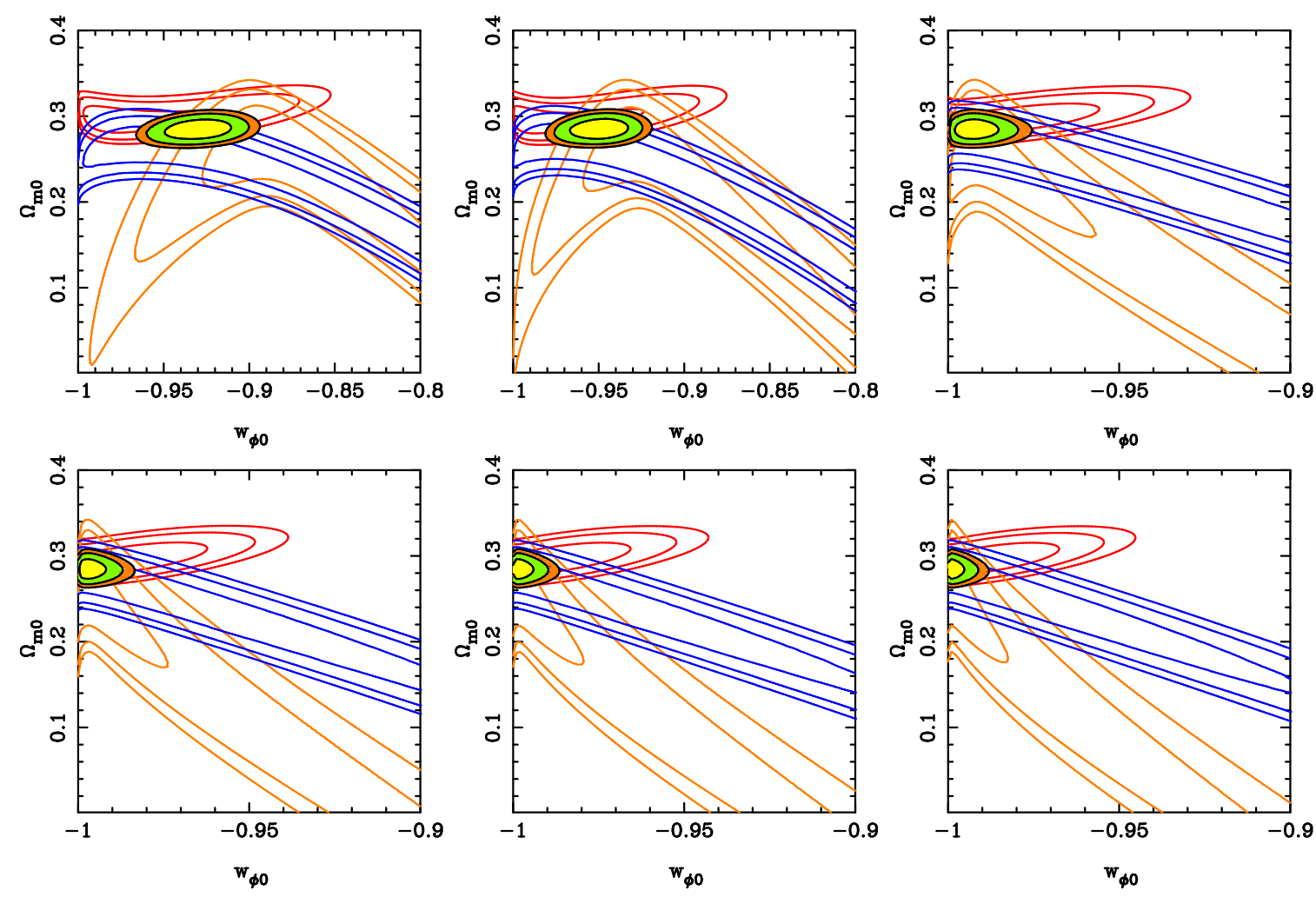

Figure 8. Contours in the $\Omega_{m 0}-w_{\phi 0}$ plane for a fixed value of $\phi_{0} / \phi_{a}=0.1$ for the exponential potential. In the plots in row- $1 \phi_{0} H_{0}=0.08,0.1$ and 0.3 from left to right and in row- $2 \phi_{0} H_{0}=0.5,0.7$ and 0.9 . The red, orange and blue colours represent $\mathrm{BAO}, \mathrm{H}(\mathrm{z})$ and $\mathrm{SN}-\mathrm{Ia}$ data respectively. Black, dark contours are for combined dataset.

In figure 2 we show the agreement between data and theory with exponential potential (2.14). We plot the acoustic parameter $A(z)$, the Hubble parameter $H(z)$ and the distance modulus $\mu(z)$ as a function of redshift $z$ along with the data points and the error bars, taken from [62, 69, 81]. There are six overlapping theoretical curves in each of these plots representing the exponential potential. To draw these theoretical curves, we have taken the best fit values of the parameters $\phi_{0} H_{0}, \Omega_{m 0}$ and $w_{\phi 0}$ from each row of table 2 .

To show the degeneracy mentioned above, we first plot the $1 \sigma, 2 \sigma$ and $3 \sigma$ contours in $w_{\phi 0}-$ $\phi_{0} H_{0}$ plane in figure 7. In these plots, we have fixed $\Omega_{m 0}=0.285$ and first, second and third row are for $\phi_{0} / \phi_{a}=0.01,0.1$ and 1.0. We can see that all datasets (BAO, Hz and SN-Ia) have lower bound on $\phi_{0} H_{0}$. The lower bound on this parameter depends on the value of parameter $\phi_{0} / \phi_{a}$; for $\phi_{0} / \phi_{a}=0.01$ we have $\phi_{0} H_{0} \gtrsim 4 \times 10^{-3}$, for $\phi_{0} / \phi_{a}=0.1$ we have $\phi_{0} H_{0} \gtrsim 0.04$ and $\phi_{0} / \phi_{a}=1.0$ we have $\phi_{0} H_{0} \gtrsim 0.41$. The lower bound on $\phi_{0} H_{0}$ increases with $\phi_{0} / \phi_{a}$. We fix the parameter $\phi_{0} / \phi_{a}=0.1$, and we do our analysis by keeping other parameters free. The analysis below is equally valid for any other value of this parameter if $\phi_{0} H_{0}$ is adjusted accordingly or properly scaled.

We have shown constraints on $\Omega_{m 0}-w_{\phi 0}$ plane in figure 8 for $\phi_{0} H_{0}=0.08,0.1,0.3,0.5,0.7$ and 0.9. Here we have fixed $\phi_{0} / \phi_{a}=0.1$. The contours filled with three different colours represent result for the combination of datasets. The $3 \sigma$ results for this model are shown in table 2 . We started from $\phi_{0} H_{0}=0.08$ as for smaller values, the value of $\chi_{\min }^{2}$ increases sharply. We can see that for a smaller value of $\phi_{0} H_{0}$ the three datasets are not in good agreement with each other and hence a large value of $\chi_{\min }^{2}$. As we increase the value of this parameter, the value of $\chi_{\min }^{2}$ decreases and the 


\begin{tabular}{|c|c|c|c|c|}
\hline$\phi_{0} H_{0}$ & $\chi_{\text {min }}^{2}$ & $\Omega_{m 0}$ & $w_{\phi 0}$ & $\frac{V_{a}}{\rho_{c r}}$ \\
\hline 0.08 & 600.125 & $0.285_{-0.022}^{+0.022}$ & $-0.928_{-0.038}^{+0.034}$ & {$[0.724,0.801]$} \\
\hline 0.10 & 595.862 & $0.285_{-0.022}^{+0.023}$ & $-0.949_{-0.032}^{+0.030}$ & {$[0.733,0.806]$} \\
\hline 0.30 & 590.327 & $0.285_{-0.022}^{+0.023}$ & $-0.993_{-0.007}^{+0.017}$ & {$[0.756,0.815]$} \\
\hline 0.50 & 590.136 & $0.284_{-0.021}^{+0.024}$ & $-0.997_{-0.003}^{+0.013}$ & {$[0.759,0.815]$} \\
\hline 0.70 & 590.132 & $0.285_{-0.022}^{+0.023}$ & $-0.999_{-0.001}^{+0.012}$ & {$[0.760,0.815]$} \\
\hline 0.90 & 590.061 & $0.285_{-0.022}^{+0.023}$ & $-0.999_{-0.001}^{+0.011}$ & {$[0.760,0.815]$} \\
\hline
\end{tabular}

Table 2. Best fit values for $\Omega_{m 0}$ and $w_{\phi 0}$ with $3 \sigma$ confidence interval for the exponential potential for different values of $\phi_{0} H_{0}$ for combine data (BAO $\left.+\mathrm{H}(\mathrm{z})+\mathrm{SN}-\mathrm{Ia}\right)$ set. Here we have fixed the value of $\phi_{0} / \phi_{a}=0.1$. In the last column, we have shown the range of amplitude of potential $V_{a}$ normalized by present critical density $\rho_{c r}$. It is calculated from equation (2.16) considering $3 \sigma$ confidence range of $\Omega_{m 0}$ and $w_{\phi 0}$.
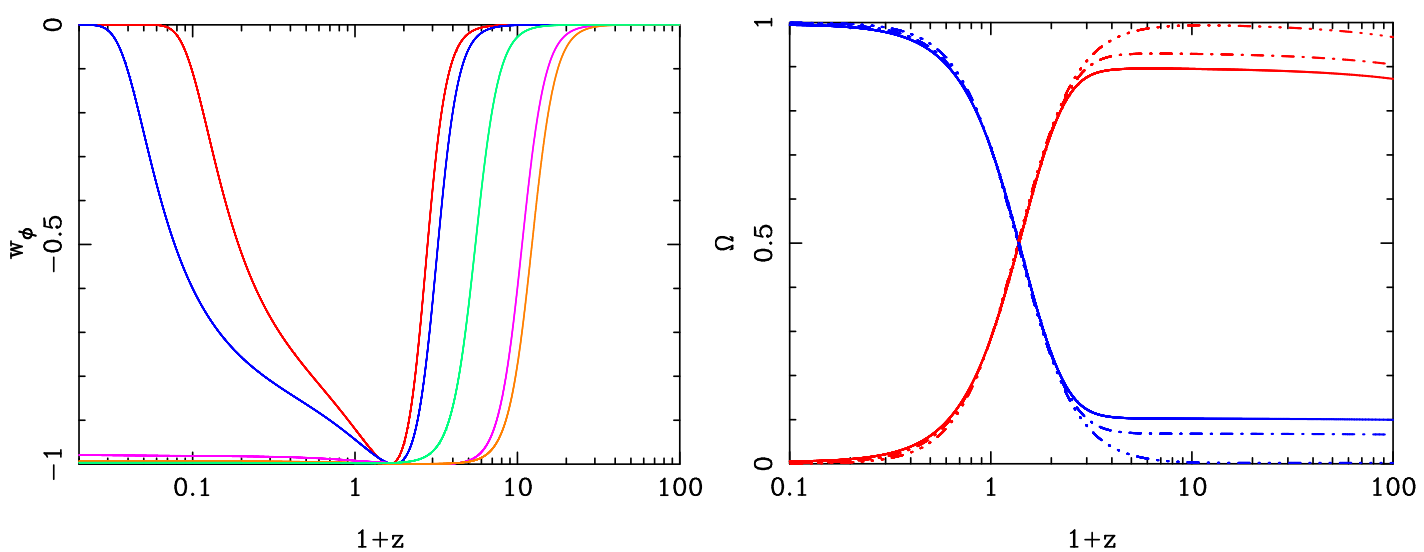

Figure 9. The plot on the left shows the evolution of equation of state $w_{\phi}$ with redshift for exponential potential. Red, blue, sky blue, orange and gray lines represent $\phi_{0} H_{0}=0.08,0.1,0.3,0.5$ and 0.7 . In the plot on the right evolution of $\Omega_{m}$ (red curves) and $\Omega_{\phi}$ (blue curves) with redshift are shown. Solid, dash-dot and dasheddot-dot-dot lines represent $\phi_{0} H_{0}=0.08,0.1$ and 0.3 . Parameter $\Omega_{m 0}$ and $w_{\phi 0}$ are the best fit values taken from table 2 for the corresponding value of $\phi_{0} H_{0}$. Parameter $\phi_{0} / \phi_{a}=0.1$.

combined contours become smaller. The BAO data provides the tightest constraint on $\Omega_{m 0}$ among all; this is consistent with previous studies [17, 18]. The value of this parameter is $\Omega_{m 0}=0.285_{-0.022}^{+0.023}$ 
with $3 \sigma$ confidence for the combination of all three datasets, and it is almost a constant with variation in $\phi_{0} H_{0}$. On the other hand, the value of $w_{\phi 0}$ depends on $\phi_{0} H_{0}$. In the last column of table 2 , we have shown the $3 \sigma$ allowed range of the amplitude of the potential normalized to the present day value of the critical density $\rho_{c r}=\frac{3 H_{0}^{2}}{8 \pi G}$ using equation (2.16), considering $3 \sigma$ confidence interval of $\Omega_{m 0}$ and $w_{\phi 0}$. From equation (2.16) it is clear that the amplitude of the potential is not explicitly dependent on $\phi_{0} H_{0}$ and as we have fixed $\phi_{0} / \phi_{a}=0.1$; its value only depends on other parameters $\Omega_{m 0}$ and $w_{\phi 0}$. Since the values of these parameters saturates with an increase in $\phi_{0} H_{0}$, the amplitude of potential also approaches a fixed value unlike the case of tachyon model with inverse square potential.

The evolution of the equation of state parameter at different epochs in the expansion history of the universe is shown in the plot on the left in figure 9. For a tachyon field with an exponential potential, the accelerating phase is sandwiched between two decelerating phases. In future, the universe goes back to a decelerating phase and duration of the accelerating phase depends on the value of $\phi_{0} H_{0}$ and $w_{\phi 0}$. In this plot, we can see that for a smaller value of $\phi_{0} H_{0}$, this duration is small and the universe goes to decelerating phase once again in relatively near future than it is for larger values of this parameter. The notable thing here is that parameters $\phi_{0} H_{0}$ and $w_{\phi 0}$ are correlated and for small $\phi_{0} H_{0}$ the best fit value of $w_{\phi 0}$ is large or away from - 1 .

We can see that in the matter dominated era, the dark energy behaves like a fluid and in the near past, it starts to deviate from $w_{\phi}=0$ sharply. For a larger value of $\phi_{0} H_{0}$, its deviation begins earlier. At first, it goes close to -1 depending on its present day value $w_{\phi 0}$ and then it rises away from -1 . For a smaller value of $\phi_{0} H_{0}$ it faster approach to a fluid like equation of state $w_{\phi}=0$ and as it crosses the condition $w_{\phi} \leq-1 / 3$ for an accelerated expansion and the universe goes to a decelerating phase once again. We have shown the evolution of density parameters $\Omega_{m}$ (red curves) and $\Omega_{\phi}$ (blue curves) with redshift in the plot on the right in figure 9. In the matter dominated era, matter does not fully dominate the energy budget. Part of sub-dominated dark energy density parameter is large (solid line) for a smaller value of $\phi_{0} H_{0}$ and as we increase the value of this parameter non-relativistic matter dominates the energy of the universe completely.

\section{Summary and Conclusions}

In this work, we have constrained parameters of the tachyon dark energy model with an inverse square potential and an exponential potential. For this purpose, we have used the Baryon acoustic Oscillation data (from SDSS DR12, 6dFGS, SDSS DR7, WingleZ surveys), direct measurement of Hubble parameter $(\mathrm{H}(\mathrm{z}))$ data and Supernova-Ia Union 2.1 data. For the inverse square potential, we have three parameters $\phi_{0} H_{0}, w_{\phi 0}$ and $\Omega_{m 0}$. For the exponential potential, apart from these three, an extra parameter $\phi_{0} / \phi_{a}$ is present. There is a lower bound on the parameter $\phi_{0} H_{0}$, and all larger values are allowed. For the inverse square potential, $\phi_{0} H_{0} \geq 0.775$ at the $3 \sigma$ confidence level. For the exponential potential, this value depends on $\phi_{0} / \phi_{a}$, and the lower bound on $\phi_{0} H_{0}$ increases with increase in $\phi_{0} / \phi_{a}$. Using combined data of all three measurements, we find that the present day matter density parameter is constrained to the values $\Omega_{m 0}=0.285_{-0.022}^{+0.023}$ at the $3 \sigma$ confidence for both the potentials and it remains almost same with variation in $\phi_{0} H_{0}$. This value of $\Omega_{m 0}$ for the tachyon model is less than the value of this parameter for a flat $\Lambda C D M$ model as determined by current observations, e.g., $\Omega_{m 0}=0.295 \pm 0.034$ ( at $68 \%$ confidence using JLA data [87]), $\Omega_{m 0}=0.3089 \pm 0.0062$ (at $68 \%$ confidence obtained from CMB-TT,TE,EE+ low-P + lensing + BAO+JLA+ $H_{0}$ data [2]), $\Omega_{m 0}=$ $0.311 \pm 0.0056$ (at $68 \%$ confidence obtained from CMB-TT,TE,EE+ low-P + lensing + BAO data [3]) and $\Omega_{m 0}=0.310 \pm 0.005$ (at 95\% confidence using BAO DR12 + SN-Ia data [83]). The value of $\Omega_{m 0}$ for tachyon model is in agreement with its value for flat $\Lambda C D M$ model constrained by the JLA data within $1 \sigma$. There is a tension with constraints from Planck and BAO DR12 data. 
The value of $w_{\phi 0}$ depends on $\phi_{0} H_{0}$. For a smaller value of $\phi_{0} H_{0}$, the equation of state parameter $w_{\phi 0}$ has a larger value and as its value increases, $w_{\phi 0}$ approaches -1 . A large range of $\phi_{0} H_{0}$ is allowed by the background data. The parameter $\phi_{0} H_{0}$ need to be tuned to obtain the value of the equation of state parameter $w_{\phi 0}$ which is supported by observations $\left(w_{\phi 0}=-1.006 \pm 0.045\right.$ [2] and $w_{\phi 0}=$ $-1.03 \pm 0.03$ [3] ). This tuning is not as severe as the fine-tuning problems in $\Lambda C D M$ model. This parameter is constrained from below to a value closer to unity, and there is no upper bound. Therefore the tuning of this parameter is not severe. The potentials, we have used in this paper, have also been extensively used for canonical scalar field (quintessence field) model of dark energy and similar results have been found [24, 25, 88-91]. Specially, tracker solutions of quintessence model are able to solve the fine-tuning problem, and thawing or freezing model ameliorate this problem $[24,25,89,91]$. In [88], it has been shown that for the potential $V(\phi) \propto \phi^{-n}$, with $n<5$, the solutions do not have finetuning problem and a large range of initial conditions provide acceptable solutions. Similar results have also been shown in [24, 25, 89, 92] for inverse power law potentials. Exponential potential have been studied in [24, 92-94] for quintessence model and it is found to ameliorate the fine-tuning problem. In our study, tachyon models with both the potentials generate acceptable solution for large range of parameters. On the other hand, for larger value of $\phi_{0} H_{0}$, it is able to mimic the cosmological constant like equation of state at present. Hence, in the light of current observational data, tachyon model is an interesting and important alternate for dark energy.

We have also studied the evolution of the phases of expansion, the density parameters and the equation of state of dark energy with redshift. We find the transition redshift to be in the range $0.61 \lesssim z_{a c} \lesssim 0.80$. For the exponential potential, the duration of the acceleration phase depends on $\phi_{0} H_{0}$ and $w_{\phi 0}$ (as these parameters are correlated). For a smaller value of $\phi_{0} H_{0}$ this duration is small. The equation of state of the tachyon dark energy, in the matter dominated and earlier phases, is dust like $\left(w_{\phi}=0\right)$. It then makes a sharp transition to that of a cosmological constant as dark energy domination begin. The value of the equation of state parameter rises again to match its present day value $w_{\phi 0}$. For the inverse square potential, it approached a constant value depending on the values of $\phi_{0} H_{0}$ and $w_{\phi 0}$. For exponential potential, it rises towards $w_{\phi}=0$, and as it becomes greater than $-1 / 3$, the universe once again goes to a decelerating expansion phase. For tachyon dark energy, matter does not fully dominate the energy budget. However, as we increase the value of parameter $\phi_{0} H_{0}$, it approaches to dominating fully as the equation of state approaches like that of a cosmological constant.

The constraints obtained here are stringent, and there is a clear preference for models which are close to the cosmological constant model. A specific set of parameters can be ruled out in a given set of models whereas current data cannot completely distinguish between different models and does not fully rule out any. The range of the combined constraints on the matter density parameter and the equation of state parameter are determined largely by the BAO data and by the supernova data respectively. While the Hubble parameter data constrains the parameters well, the allowed range is larger than that allowed by other observations. This is possibly due to the fact that the Hubble parameter measurement data is a compilation of measurements with different methods and accompanies measurements of different cosmological quantities. The constraints on the parameters are stringent and more data, and further studies in perturbations in tachyon dark energy are likely to break the degeneracy between different models which are allowed by pure distance measurements.

\section{Acknowledgements}

The numerical work in this paper was done using the High Performance Computing facility at IISER Mohali. The authors thank J. S. Bagla and Ankan Mukherjee for helpful discussions. 


\section{References}

[1] Planck collaboration, P. A. R. Ade et al., Planck 2013 results. I. Overview of products and scientific results, Astron. Astrophys. 571 (2014) A1 [1303.5062].

[2] Planck Collaboration, Ade, P. A. R., Aghanim, N., Arnaud, M., Ashdown, M., Aumont, J. et al., Planck 2015 results - xiii. cosmological parameters, A\&A 594 (2016) A13.

[3] Planck Collaboration, N. Aghanim, Y. Akrami, M. Ashdown, J. Aumont, C. Baccigalupi et al., Planck 2018 results. VI. Cosmological parameters, arXiv e-prints (2018) arXiv:1807.06209 [1807 . 06209 ].

[4] S. Perlmutter, S. Gabi, G. Goldhaber, A. Goobar, D. E. Groom, I. M. Hook et al., Measurements of the cosmological parameters $\Omega$ and $\Lambda$ from the first seven supernovae at $z \leq 0.35$, The Astrophysical Journal 483 (1997) 565.

[5] S. Perlmutter, G. Aldering, G. Goldhaber, R. A. Knop, P. Nugent, P. G. Castro et al., Measurements of $\Omega$ and $\Lambda$ from 42 high-redshift supernovae, The Astrophysical Journal 517 (1999) 565.

[6] A. G. Riess, A. V. Filippenko, P. Challis, A. Clocchiatti, A. Diercks, P. M. Garnavich et al., Observational evidence from supernovae for an accelerating universe and a cosmological constant, The Astronomical Journal 116 (1998) 1009.

[7] S. M. Carroll, W. H. Press and E. L. Turner, The cosmological constant, Annual Review of Astronomy and Astrophysics 30 (1992) 499.

[8] S. M. Carroll, The Cosmological constant, Living Rev. Rel. 4 (2001) 1 [astro-ph/0 004075 ].

[9] M. Chevallier and D. Polarski, Accelerating Universes with Scaling Dark Matter, International Journal of Modern Physics D 10 (2001) 213 [gr-qc/ 000900 8].

[10] E. V. Linder, Exploring the expansion history of the universe, Phys. Rev. Lett. 90 (2003) 091301.

[11] H. K. Jassal, J. S. Bagla and T. Padmanabhan, Observational constraints on low redshift evolution of dark energy: How consistent are different observations?, Phys. Rev. D 72 (2005) 103503.

[12] G. Efstathiou, Constraining the equation of state of the universe from distant type ia supernovae and cosmic microwave background anisotropies, Monthly Notices of the Royal Astronomical Society 310 (1999) 842.

[13] S. Lee, Constraints on the dark energy equation of state from the separation of cmb peaks and the evolution of $\alpha$, Phys. Rev. D 71 (2005) 123528.

[14] S. Hannestad and E. Mrtsell, Cosmological constraints on the dark energy equation of state and its evolution, Journal of Cosmology and Astroparticle Physics 2004 (2004) 001.

[15] Y. Wang and M. Tegmark, New dark energy constraints from supernovae, microwave background, and galaxy clustering, Phys. Rev. Lett. 92 (2004) 241302.

[16] D. Huterer and M. S. Turner, Probing dark energy: Methods and strategies, Phys. Rev. D 64 (2001) 123527.

[17] A. Tripathi, A. Sangwan and H. K. Jassal, Dark energy equation of state parameter and its evolution at low redshift, JCAP 1706 (2017) 012 [1611.01899].

[18] A. Sangwan, A. Mukherjee and H. K. Jassal, Reconstructing the dark energy potential, JCAP 1801 (2018) 018 [1712.05143].

[19] W. Zheng and H. Li, Constraints on parameterized dark energy properties from new observations with principal component analysis, Astroparticle Physics 86 (2017) 1.

[20] S. Kumar and L. Xu, Observational constraints on variable equation of state parameters of dark matter and dark energy after planck, Physics Letters B 737 (2014) 244 .

[21] M. Rezaei, M. Malekjani, S. Basilakos, A. Mehrabi and D. F. Mota, Constraints to dark energy using pade parameterizations, The Astrophysical Journal 843 (2017) 65. 
[22] W. Yang, S. Pan and A. Paliathanasis, Latest astronomical constraints on some non-linear parametric dark energy models, Monthly Notices of the Royal Astronomical Society 475 (2018) 2605.

[23] S. Cao and Z.-H. Zhu, Cosmic equation of state from combined angular diameter distances: Does the tension with luminosity distances exist?, Phys. Rev. D 90 (2014) 083006.

[24] B. Ratra and P. J. E. Peebles, Cosmological Consequences of a Rolling Homogeneous Scalar Field, Phys. Rev. D37 (1988) 3406.

[25] E. V. Linder, The Dynamics of Quintessence, The Quintessence of Dynamics, Gen. Rel. Grav. 40 (2008) 329 [0704.2064].

[26] D. Huterer and H. V. Peiris, Dynamical behavior of generic quintessence potentials: Constraints on key dark energy observables, Phys. Rev. D75 (2007) 083503 [astro-ph / 0610427 ].

[27] I. Zlatev, L.-M. Wang and P. J. Steinhardt, Quintessence, cosmic coincidence, and the cosmological constant, Phys. Rev. Lett. 82 (1999) 896 [astro-ph/9807002].

[28] E. J. Copeland, A. R. Liddle and D. Wands, Exponential potentials and cosmological scaling solutions, Phys. Rev. D57 (1998) 4686 [gr-qc/9711068].

[29] A. Sangwan, A. Tripathi and H. K. Jassal, Observational constraints on quintessence models of dark energy, 1804.09350.

[30] C. R. Watson and R. J. Scherrer, The Evolution of inverse power law quintessence at low redshift, Phys. Rev. D68 (2003) 123524 [astro-ph / 0306364 ].

[31] R. J. Scherrer and A. A. Sen, Thawing quintessence with a nearly flat potential, Phys. Rev. D77 (2008) 083515 [0712.3450].

[32] S. Dutta and R. J. Scherrer, Slow-roll freezing quintessence, Phys. Lett. B704 (2011) 265 [1106.0012].

[33] Y. Chen, C.-Q. Geng, S. Cao, Y.-M. Huang and Z.-H. Zhu, Constraints on a $\phi c d m$ model from strong gravitational lensing and updated hubble parameter measurements, Journal of Cosmology and Astroparticle Physics 2015 (2015) 010.

[34] J. S. Bagla, H. K. Jassal and T. Padmanabhan, Cosmology with tachyon field as dark energy, Phys. Rev. D 67 (2003) 063504.

[35] A. Sen, Rolling tachyon, Journal of High Energy Physics 2002 (2002) 048.

[36] A. Sen, Tachyon matter, Journal of High Energy Physics 2002 (2002) 065.

[37] A. SEN, Field theory of tachyon matter, Modern Physics Letters A 17 (2002) 1797.

[38] T. Padmanabhan, Accelerated expansion of the universe driven by tachyonic matter, Phys. Rev. D 66 (2002) 021301.

[39] G. Calcagni and A. R. Liddle, Tachyon dark energy models: Dynamics and constraints, Phys. Rev. D 74 (2006) 043528.

[40] E. J. Copeland, M. R. Garousi, M. Sami and S. Tsujikawa, What is needed of a tachyon if it is to be the dark energy?, Phys. Rev. D 71 (2005) 043003.

[41] J. M. Aguirregabiria and R. Lazkoz, Tracking solutions in tachyon cosmology, Phys. Rev. D 69 (2004) 123502.

[42] M. Fairbairn and M. H. Tytgat, Inflation from a tachyon fluid?, Physics Letters B 546 (2002) 1.

[43] L. Kofman and A. Linde, Problems with tachyon inflation, Journal of High Energy Physics 2002 (2002) 004.

[44] A. Feinstein, Power-law inflation from the rolling tachyon, Phys. Rev. D 66 (2002) 063511.

[45] K. Rezazadeh, K. Karami and S. Hashemi, Tachyon inflation with steep potentials, Phys. Rev. D 95 (2017) 103506. 
[46] N. Barbosa-Cendejas, J. De-Santiago, G. German, J. C. Hidalgo and R. R. Mora-Luna, Tachyon inflation in the large- $n$ formalism, Journal of Cosmology and Astroparticle Physics 2015 (2015) 020.

[47] Q. Fei, Y. Gong, J. Lin and Z. Yi, The reconstruction of tachyon inflationary potentials, Journal of Cosmology and Astroparticle Physics 2017 (2017) 018.

[48] Q. Gao, Y. Gong and Q. Fei, Constant-roll tachyon inflation and observational constraints, Journal of Cosmology and Astroparticle Physics 2018 (2018) 005.

[49] N. Barbosa-Cendejas, J. De-Santiago, G. German, J. C. Hidalgo and R. R. Mora-Luna, Theoretical and observational constraints on tachyon inflation, Journal of Cosmology and Astroparticle Physics 2018 (2018) 015.

[50] T. Padmanabhan and T. R. Choudhury, Can the clustered dark matter and the smooth dark energy arise from the same scalar field?, Phys. Rev. D 66 (2002) 081301.

[51] S. Sugimoto and S. Terashima, Tachyon matter in boundary string field theory, Journal of High Energy Physics 2002 (2002) 025.

[52] A. Das and A. DeBenedictis, Inhomogeneous cosmologies with tachyonic dust as dark matter, General Relativity and Gravitation 36 (2004) 1741.

[53] P. C. W. Davies, Tachyonic dark matter, International Journal of Theoretical Physics 43 (2004) 141.

[54] M. A. Makukov, E. G. Mychelkin and V. L. Saveliev, On possible tachyonic state of neutrino dark matter, International Journal of Modern Physics: Conference Series 41 (2016) 1660133 [https://www.worldscientific.com/doi/pdf/10.1142/S2010194516601332].

[55] H.-J. Seo and D. J. Eisenstein, Probing dark energy with baryonic acoustic oscillations from future large galaxy redshift surveys, The Astrophysical Journal 598 (2003) 720.

[56] W. J. Percival, R. C. Nichol, D. J. Eisenstein, J. A. Frieman, M. Fukugita, J. Loveday et al., The shape of the sloan digital sky survey data release 5 galaxy power spectrum, The Astrophysical Journal 657 (2007) 645.

[57] Busca, N. G., Delubac, T., Rich, J., Bailey, S., Font-Ribera, A., Kirkby, D. et al., Baryon acoustic oscillations in the lyest of boss quasars, A\&A 552 (2013) A96.

[58] C. Blake, S. Brough, M. Colless, C. Contreras, W. Couch, S. Croom et al., The wigglez dark energy survey: joint measurements of the expansion and growth history at $z<1$, Monthly Notices of the Royal Astronomical Society 425 (2012) 405.

[59] L. Anderson, . Aubourg, S. Bailey, F. Beutler, V. Bhardwaj, M. Blanton et al., The clustering of galaxies in the SDSS - II baryon oscillation spectroscopic survey: baryon acoustic oscillations in the data releases 10 and 11 galaxy samples, Monthly Notices of the Royal Astronomical Society 441 (2014) 24.

[60] A. Veropalumbo, F. Marulli, L. Moscardini, M. Moresco and A. Cimatti, An improved measurement of baryon acoustic oscillations from the correlation function of galaxy clusters at $z \sim 0.3$, Monthly Notices of the Royal Astronomical Society 442 (2014) 3275.

[61] Delubac, Timothée, Bautista, Julian E., Busca, Nicolás G., Rich, James, Kirkby, David, Bailey, Stephen et al., Baryon acoustic oscillations in the lyest of boss DR11 quasars, A\&A 574 (2015) A59.

[62] N. Suzuki, D. Rubin, C. Lidman, G. Aldering, R. Amanullah, K. Barbary et al., The hubble space telescope cluster supernova survey. $v$. improving the dark-energy constraints above $Z>1$ and building an early-type-hosted supernova sample, The Astrophysical Journal 746 (2012) 85.

[63] P. M. Garnavich, S. Jha, P. Challis, A. Clocchiatti, A. Diercks, A. V. Filippenko et al., Supernova limits on the cosmic equation of state, The Astrophysical Journal 509 (1998) 74.

[64] J. L. Tonry, B. P. Schmidt, B. Barris, P. Candia, P. Challis, A. Clocchiatti et al., Cosmological results from high-z supernovae, The Astrophysical Journal 594 (2003) 1. 
[65] B. J. Barris, J. L. Tonry, S. Blondin, P. Challis, R. Chornock, A. Clocchiatti et al., Twenty-three high-redshift supernovae from the institute for astronomy deep survey: Doubling the supernova sample at $z>0.7$, The Astrophysical Journal 602 (2004) 571.

[66] A. Goobar, S. Perlmutter, G. Goldhaber, R. A. Knop, P. Nugent, P. G. Castro et al., The acceleration of the universe: Measurements of cosmological parameters from type ia supernovae, Physica Scripta 2000 (2000) 47.

[67] S. Gonzlez-Gaitn, A. Conley, F. B. Bianco, D. A. Howell, M. Sullivan, K. Perrett et al., The rise time of normal and subluminous type ia supernovae, The Astrophysical Journal 745 (2012) 44.

[68] Astier, P., Guy, J., Regnault, N., Pain, R., Aubourg, E., Balam, D. et al., The supernova legacy survey: measurement of $\Omega_{m}, \Omega_{\Lambda}$ and $w$ from the first year data set the first year data set, A\&A 447 (2006) 31 .

[69] O. Farooq, F. R. Madiyar, S. Crandall and B. Ratra, Hubble parameter measurement constraints on the redshift of the deceleration-acceleration transition, dynamical dark energy, and space curvature, The Astrophysical Journal 835 (2017) 26.

[70] O. Farooq and B. Ratra, Hubble parameter measurement constraints on the cosmological deceleration-acceleration transition redshift, The Astrophysical Journal Letters 766 (2013) L7.

[71] O. Farooq, D. Mania and B. Ratra, Hubble parameter measurement constraints on dark energy, The Astrophysical Journal 764 (2013) 138.

[72] L. Samushia and B. Ratra, Cosmological constraints from hubble parameter versus redshift data, The Astrophysical Journal Letters 650 (2006) L5.

[73] D. Stern, R. Jimenez, L. Verde, M. Kamionkowski and S. A. Stanford, Cosmic chronometers: constraining the equation of state of dark energy. $i: H(z)$ measurements, Journal of Cosmology and Astroparticle Physics 2010 (2010) 008.

[74] M. Moresco, A. Cimatti, R. Jimenez, L. Pozzetti, G. Zamorani, M. Bolzonella et al., Improved constraints on the expansion rate of the universe up to $z \sim 1.1$ from the spectroscopic evolution of cosmic chronometers, Journal of Cosmology and Astroparticle Physics 2012 (2012) 006.

[75] C.-H. Chuang and Y. Wang, Modelling the anisotropic two-point galaxy correlation function on small scales and single-probe measurements of $H(z), D A(z)$ and $f(z) \sigma 8(z)$ from the sloan digital sky survey DR7 luminous red galaxies, Monthly Notices of the Royal Astronomical Society 435 (2013) 255.

[76] Y. Chen and B. Ratra, Hubble parameter data constraints on dark energy, Physics Letters B $\mathbf{7 0 3}$ (2011) 406 .

[77] T. Barreiro, B. d. Carlos and E. J. Copeland, Nonperturbative corrections to the kähler potential, Phys. Rev. D 57 (1998) 7354.

[78] P. Bintruy, Models of dynamical supersymmetry breaking and quintessence, Phys. Rev. D 60 (1999) 063502.

[79] F. Rosati, Quintessential enhancement of dark matter abundance, Physics Letters B $\mathbf{5 7 0}$ (2003) 5 .

[80] T. Ngampitipan and P. Wongjun, Dynamics of three-form dark energy with dark matter couplings, Journal of Cosmology and Astroparticle Physics 2011 (2011) 036.

[81] C. Blake, E. A. Kazin, F. Beutler, T. M. Davis, D. Parkinson, S. Brough et al., The wigglez dark energy survey: mapping the distanceredshift relation with baryon acoustic oscillations, Monthly Notices of the Royal Astronomical Society 418 (2011) 1707.

[82] Y. Wang and S. Wang, Distance priors from planck and dark energy constraints from current data, Phys. Rev. D 88 (2013) 043522.

[83] S. Alam, M. Ata, S. Bailey, F. Beutler, D. Bizyaev, J. A. Blazek et al., The clustering of galaxies in the completed SDSS - II baryon oscillation spectroscopic survey: cosmological analysis of the DR 12 galaxy sample, Monthly Notices of the Royal Astronomical Society 470 (2017) 2617. 
[84] BOSS Collaboration collaboration, E. Aubourg, S. Bailey, J. E. Bautista, F. Beutler, V. Bhardwaj, D. Bizyaev et al., Cosmological implications of baryon acoustic oscillation measurements, Phys. Rev. D 92 (2015) 123516.

[85] C.-H. Chuang, M. Pellejero-Ibanez, S. Rodrguez-Torres, A. J. Ross, G.-b. Zhao, Y. Wang et al., The clustering of galaxies in the completed SDSS - III baryon oscillation spectroscopic survey: single-probe measurements from DR12 galaxy clustering towards an accurate model, Monthly Notices of the Royal Astronomical Society 471 (2017) 2370.

[86] D. J. Eisenstein, I. Zehavi, D. W. Hogg, R. Scoccimarro, M. R. Blanton, R. C. Nichol et al., Detection of the baryon acoustic peak in the large-scale correlation function of SDSS luminous red galaxies, The Astrophysical Journal 633 (2005) 560.

[87] Betoule, M., Kessler, R., Guy, J., Mosher, J., Hardin, D., Biswas, R. et al., Improved cosmological constraints from a joint analysis of the SDSS - II and SNLS supernova samples, A\&A 568 (2014) A22.

[88] A. de la Macorra and C. Stephan-Otto, Quintessence restrictions on negative power and condensate potentials, Phys. Rev. D 65 (2002) 083520.

[89] I. Zlatev, L. Wang and P. J. Steinhardt, Quintessence, cosmic coincidence, and the cosmological constant, Phys. Rev. Lett. 82 (1999) 896.

[90] A. Riazuelo and J.-P. Uzan, Quintessence and gravitational waves, Phys. Rev. D 62 (2000) 083506.

[91] T. Matos and L. Arturo Ureña López, Further analysis of a cosmological model with quintessence and scalar dark matter, Phys. Rev. D 63 (2001) 063506.

[92] A. A. Sen, G. Gupta and S. Majumdar, Constraining thawing quintessence, Monthly Notices of the Royal Astronomical Society 420 (2012) 1309

[http: / / oup.prod.sis.lan/mnras/article-pdf/420/2/1309/3067046/mnras0420-1309.pdf].

[93] P. G. Ferreira and M. Joyce, Structure formation with a self-tuning scalar field, Phys. Rev. Lett. 79 (1997) 4740.

[94] C. Wetterich, An asymptotically vanishing time-dependent cosmological "constant"., Astron. Astrophys. 301 (1995) 321 [hep-th/9408025]. 